\title{
Tibolone Reverses NAFLD in Ovariectomised Rats by Reducing Adiposity and Insulin Resistance
}

Lilian Brites Campos-Shimada ${ }^{1,3}$, Eduardo Hideo Gilglioni ${ }^{1}$, Danielle Aparecida Munhos Hermoso ${ }^{1}$, Ana Julia dos Reis Buzzo ${ }^{1}$, Rosângela Fernandes Garcia ${ }^{2}$, Elismari Rizato Martins-Maciel ${ }^{1}$, Emy Luiza Ishii-Iwamoto ${ }^{1}$ and Clairce Luzia Salgueiro-Pagadigorria ${ }^{2}$

1. Laboratory of Experimental Steatosis, Department of Biochemistry, State University of Maringá, Maringá 87020900, Brazil

2. Department of Physiological Sciences, State University of Maringá, Maringá 87020900, Brazil

3. Department of Biomedicine, Centro Universitário Integrado, Campo Mourão 87300970, Brazil

\begin{abstract}
This study aimed to investigate the effects of tibolone, a synthetic steroid, on several metabolic dysfunctions induced by oestrogen deficiency, in rats. Ovariectomised (OVX) rats were used as animal model of postmenopausal metabolic syndrome. The OVX rats were treated with daily doses of tibolone $(0.16 \mathrm{mg} / \mathrm{kg}$ ) and the results were compared with control (sham-operated) and OVX untreated rats. Tibolone reduced the adiposity and the visceral adipocyte size in OVX rats. The insulin sensitivity was also improved, and a decrease in the activity of the adipose tissue hormone-sensitive lipase enzyme was recorded. The lower lipolysis by visceral adipocytes, associated with the recovery of peroxisomal $\beta$-oxidation by tibolone may have contributed to the reversion of NAFLD in treated OVX rats. The reduction of liver lipid contents resulted in a general improvement in the liver redox state. In addition, tibolone reduced the mitochondrial ROS generation and restored the activity of glucose-6-phosphate dehydrogenase. Tibolone also exerted antioxidant effects on inguinal adipose tissue. Tibolone exerted several beneficial effects on cellular and metabolic dysfunctions induced by ovariectomy in rats. One important mode of action of tibolone was the reduction of the visceral adipocyte size, corroborating the relationship between this one and the development and progression of several comorbidities associated with metabolic syndrome.
\end{abstract}

Key words: Tibolone, ovariectomy, hepatic steatosis, obesity, oxidative stress.

\section{Introduction}

The postmenopausal period is commonly associated with a higher incidence of metabolic syndrome (MS), a group of metabolic and cardiovascular disturbances including visceral adiposity, insulin resistance (IR), dyslipidaemia, cardiovascular diseases and non-alcoholic fatty liver disease (NAFLD) [1]. The frequent association of these co-morbidities suggests that one or a reduced number of factors may underlie the appearance and complications of all these disorders [2].

In this context, visceral adiposity has been considered as an independent, determinant factor in the

Corresponding author: Lilian Brites Campos-Shimada, $\mathrm{Ph} . \mathrm{D}$., professor, research fields: metabolism and oxidative stress. development of most complications associated with obesity, such as IR and NAFLD [2]. The hypertrophy of adipocytes induces important cellular dysfunctions, including over-secretion of tumour necrosis factor-alpha (TNF- $\alpha)$, leptin and other pro-inflammatory adipokines and diminished secretion of adiponectin [3-7]. At the cellular level, TNF- $\alpha$-dependent activation of stress-related kinases inhibits insulin signal transduction, causing IR in both adipocytes and myocytes [8]. Insulin resistance, in turn, represents the "first-level" event, responsible for the development of several complications in obesity or "IR syndromes", such as NAFLD [9, 10].

In addition, adipocytes secrete C-reactive protein and other cytokines, such as leptin and interleukin-6, which similarly to $\mathrm{TNF}-\alpha$, are pro-inflammatory $[3,4$, 
9]. Thus, the development of a chronic low-grade inflammatory state represents the "second-level" contributing event for the pathogenesis of the metabolic and cardiovascular complications in obesity $[2,7]$.

Hormone replacement therapy (HRT) may improve many factors associated with MS, including fat mass and distribution, dyslipidaemia, IR and NAFLD [1, 11]. However, the time of exposure and initiation of postmenopausal oestrogen therapy may be an important factor in the development of cardiovascular diseases [12].

Tibolone is a synthetic steroid used as an alternative form of HRT and, similarly to conventional steroids, exerts positive effects on several abnormalities of the MS, including the prevention of weight gain both in postmenopausal women and in animal models of oestrogen deficiency $[13,14]$.

Rodent ovariectomy (OVX) is an approach used to model human menopause and to study the metabolic consequences of loss of ovarian function. Studies have demonstrated that, 13 weeks after surgical ovary removal, OVX rats develop obesity, IR and NAFLD [15]. Therefore, the purpose of this work was to evaluate the effects of tibolone on adiposity, plasma lipid profile, liver lipid metabolism and redox state in OVX rats. This study could also aid to clarify the relationship among adiposity, IR and NAFLD.

\section{Materials and Methods}

\subsection{Materials}

Substrates and reagents were purchased from Sigma Chemical Co. (Missouri, USA). Crystalline tibolone was obtained from New Cham S.P.A. (Italy). Kits from Gold Analisa ${ }^{\circledR}$ (Belo Horizonte, Brazil) were used for plasma biochemical analysis and to evaluate liver lipids levels. Insulin levels were measured using a Rat/Mouse Insulin ELISA Kit from Millipore (Missouri, USA). The levels of non-esterified free fatty acids (NEFA) in the serum were determined using a NEFA-HR (2) kit from Wako Chemical ${ }^{\circledR}$ (Osaka,
Japan). The lipase activity was evaluated using a Lipase Activity Colorimetric Assay Kit II from BioVision (California, USA). All reagent-grade chemicals were obtained at the highest available grade.

\subsection{Animals}

Female Wistar rats (60 days old) weighing 160 to $180 \mathrm{~g}$ were obtained from the Central Bioterium of the University of Maringá. The animals were housed in polypropylene cages (maximum of four animals per cage) and had free access to standard rodent diet $\left(\right.$ Nuvilab $^{\circledR}$, São Paulo, Brazil) and tap water. Food consumption and animal weights were recorded throughout the entire experimental period (16 weeks). Animals were housed under controlled light $(12 \mathrm{~h}$ light:12 h dark cycle starting at 06:00 h), humidity and room temperature $\left(20-23{ }^{\circ} \mathrm{C}\right)$. All experiments were conducted in strict adherence to the guidelines of the Ethics Committee for Animal Experimentation of the University of Maringá (certified 149/2014).

\subsection{Surgical Procedures and Animal Treatment}

Two days after their arrival at the laboratory, batches of female Wistar rats were randomly designated for surgical procedures of sham-operation (control) or ovariectomy (OVX). For surgery, OVX rats were anesthetized with an i.p. injection of $10 \mathrm{mg}$ xylazine + ketamine $50 \mathrm{mg} / \mathrm{kg}$ and had their ovaries removed by bilateral ovariectomy. Sham-operated (control) rats underwent the same surgical procedure but removal of the ovaries was omitted.

Thirteen weeks after ovary removal, the OVX rats were subdivided into two groups: untreated ovariectomised rats (OVX) and OVX rats treated with daily doses of tibolone $(0.16 \mathrm{mg} / \mathrm{kg}$ per day: OVX $+\mathrm{T})$. The dosage of tibolone was chosen based on preliminary dose-response studies of the beneficial effects of tibolone on the most important hepatic parameters according to the aim of this work (data not shown). The results revealed that from $0.16 \mathrm{mg} / \mathrm{kg}$ tibolone was effective in reducing the liver fat 
accumulation and improving the general oxidative state of the liver.

Tibolone was administered as a suspension in an aqueous solution of $1.0 \%$ gum arabic, by oral gavage over a period of 21 days. The OVX and sham-operated groups (control) received daily similar volumes of Arabic gum (vehicle). After this period (16 weeks after the surgical procedure), the animals were anesthetized with pentobarbital sodium $(50 \mathrm{mg} / \mathrm{kg}$ i.p.) for blood collection and removal of liver, adipose tissue and uterus.

\subsection{Sample Collection Process}

Blood was collected from fasted rats by cardiac puncture to obtain serum and plasma. The uterus was collected and weighed, and the results were expressed in $\mathrm{g}$ per $100 \mathrm{~g}$ of BW. Uterine atrophy was used as a marker for a well-established condition of oestrogen deficiency.

The liver samples were removed and, depending on the protocol, were used freshly or frozen in liquid nitrogen and stored at $-80{ }^{\circ} \mathrm{C}$. The retroperitoneal, uterine, mesenteric and inguinal fat deposits were weighed and expressed in $\mathrm{g}$ per $100 \mathrm{~g}$ of body weight (BW). The ratio between the sums of the weights of these tissues per $100 \mathrm{~g}$ of $\mathrm{BW}$ was defined as the adiposity index. The retroperitoneal and inguinal fat deposits were also frozen in liquid nitrogen for measurements of oxidative stress and hormone-sensitive lipase (HSL) activity.

\subsection{Blood Biochemical Dosages}

Total cholesterol, high-density lipoprotein (HDL cholesterol), triacylglycerols (TAG) and non-esterified free fatty acids (NEFA) were analysed in the serum, and glucose was analysed in the plasma by standard methods using assay kits. The levels of very-low-density lipoprotein (VLDL cholesterol) were calculated by Friedewald's equation, and low-density lipoprotein (LDL cholesterol) levels were determined by subtracting HDL and VLDL cholesterol from total cholesterol. The fasting plasma insulin dosages were assessed by an ELISA assay. Insulin resistance was measured in terms of the homeostasis model assessment (HOMA) index [16] using the following formula:

Homa index $=$ fasting plasm insulin $(\mathrm{mU} / \mathrm{L})$

$\times$ [fasting plasm glucose $(\mathrm{mmol}$

/ L) / 22.5]

\subsection{Adipocyte Isolation and Adipocyte Cell Size}

Adipocytes were isolated from retroperitoneal fat deposits according to the method described by Rodbell [17]. The isolated adipocytes were fixed in $4 \%$ formaldehyde in phosphate buffer saline, and images were captured with a high resolution digital camera (Pro-Series Media Cibertecnics®) coupled to an optical microscope Olympus BX40 ${ }^{\circledR}$. Image-Pro-Plus $4.1{ }^{\circledR}$ was used to measure the area of 200 adipocytes. The images were used to calculate the frequency of distribution of adipocyte sizes and their average sizes.

\subsection{Hormone-Sensitive Lipase Activity in Adipose Tissue}

Hormone-sensitive lipase (HSL) activity was evaluated in retroperitoneal and inguinal fat deposits using a Lipase Activity Colorimetric Assay Kit II from BioVision (California, USA) according to the manufacturer's instructions.

\subsection{Liver Lipid Contents and Histochemical Analysis}

Total lipid, TAG and total cholesterol liver contents were quantified after extraction of total lipids by homogenization of liver fragments in a chloroform-methanol mixture (2:1) following the method described by Folch [18]. After drying, the organic phase was weighed for determination of the total lipid contents, and afterwards, the pellet was resuspended in $200 \mu \mathrm{L}$ of $2 \%$ Triton for quantifying the content of TAG and total cholesterol, using enzymatic assay kits.

Histochemical analyses were conducted by sampling in each batch of animals to prove the existence of 
steatosis. These fragments were processed in semi-serial histological sections (10 $\mu \mathrm{m}$ thick) using a cryostat (Leica ${ }^{\circledR}$ CM1850) and stained with Sudan III, which specifically detects lipids.

\subsection{Isolation of Liver Compartments}

Liver mitochondria were isolated according to the method described by Bracht et al. [19]. The suspensions of intact mitochondria were used for ROS generation, mitochondrial $\beta$-oxidation, carbonylated protein content and nicotinamide nucleotide transhydrogenase (NNT) activity measurements. The supernatant was reserved to obtain the cytosolic fraction, as described below.

Freeze-thaw disrupted mitochondria were used for assays of reduced glutathione (GSH) levels. Mitochondria disrupted by sonication were used to measure the activities of the mitochondrial antioxidant enzymes, glutathione peroxidase 1 (GPx1), glutathione reductase (mGR) and manganese superoxide dismutase (MnSOD).

The post-mitochondrial supernatant was centrifuged at $15,000 \times \mathrm{g}$ for $10 \mathrm{~min}$ to eliminate contaminations by peroxisomes and plasma membrane vesicles. Finally, the supernatant was centrifuged at $105,000 \times \mathrm{g}$ for 60 min to precipitate microsomes. The final supernatant was the cytosolic fraction and was used to evaluate the following antioxidant enzyme activities: glutathione peroxidase (GPx3), glutathione reductase (GR) and $\mathrm{Cu}, \mathrm{ZnSOD}$.

Peroxisomes were isolated according to the method described by Natarajan et al. [20] to measure catalase (CAT) and peroxisomal fatty acyl-CoA oxidase activities (peroxisomal $\beta$-oxidation).

Total liver homogenates obtained from freeze-clamped liver of overnight fasted rats were used for GSH content and TBARS (thiobarbituric acid-reactive substances) measurements.

\subsection{Protein Determination}

Protein concentrations were determined according to the method of Lowry et al. [21] using bovine serum albumin as a standard.

\subsection{Mitochondrial and Peroxisomal $\beta$-Oxidation}

Mitochondrial fatty acid oxidation was measured polarographically at $37{ }^{\circ} \mathrm{C}$, using a Teflon-shielded platinum electrode [22]. Medium-chain (octanoate) and long-chain (palmitate) fatty acids were used in these assays as acyl-CoA derivatives in the presence of L-carnitine, and the rate of oxygen consumption was expressed as $\mathrm{nmol} / \mathrm{min} \times \mathrm{mg}$ of mitochondrial protein. The peroxisomal fatty acyl-CoA oxidase activity was measured fluorometrically [23] by the oxidation of DCFH-DA by $\mathrm{H}_{2} \mathrm{O}_{2}$ generated during the oxidation of palmitoyl-CoA in a highly fluorescent compound (DCF). The increase in fluorescence (excitation, 503 $\mathrm{nm}$; emission, $529 \mathrm{~nm}$ ) was recorded over a period of $10 \mathrm{~min}$, and the activity of fatty acid acyl-CoA oxidase was expressed as pmol DCF produced/min $\times$ mg of peroxisomal protein.

\subsection{Mitochondrial Reactive Oxygen Species (ROS) Generation}

The mitochondrial ROS generation was monitored by the oxidation of DCFH-DA by reactive oxygen species and was expressed as pmol DCF produced/min $\times \operatorname{mg}$ of mitochondrial protein [24].

\subsection{Determination of GSH, TBARS and Carbonylated} Protein Contents in the Liver

The GSH levels in liver homogenates and freeze-thaw disrupted mitochondria were measured fluorometrically according to the method described by Hissin \& Hilf [25] and expressed as $\mu \mathrm{g}$ GSH/mg protein.

The carbonylated protein contents were determined in liver homogenates and freshly mitochondria as described by Hermoso et al. [26]. The carbonyl group levels were calculated using the molar absorption coefficient for aliphatic hydrazones at $370 \mathrm{~nm}$ of $22,000 \mathrm{M}^{-1} \mathrm{~cm}^{-1}$ and expressed as nmol carbonyl $/ \mathrm{mg}$ 
protein.

Lipid peroxidation levels were measured spectrophotometrically by the TBARS method [27] in liver homogenates, and the results were expressed as nmol of malondialdehyde (MDA)/mg protein using a molar extinction coefficient for MDA of $1.56 \times 10^{5} \mathrm{M}^{-1}$ $\mathrm{cm}^{-1}$

\subsection{Hepatic Antioxidant Enzyme Activities}

CAT activity was measured in isolated peroxisomes according to the method described by Aebi [28]. Glucose-6 phosphate dehydrogenase (G6PD) activity was measured in the $30,000 \times \mathrm{g}$ supernatants obtained from homogenates of freeze clamped livers in $0.1 \mathrm{M}$ Tris/HCl buffer [29].

The activities of other antioxidant enzymes were measured in the cytosolic and mitochondrial fractions. Glutathione peroxidase (GPx1 and GPx3) activity was measured by the rate of NADPH oxidation in the presence of $\mathrm{H}_{2} \mathrm{O}_{2}$ [30]. Glutathione reductase (GR) activity was determined spectrophotometrically by decreases in NADPH concentrations in the presence of oxidized glutathione (GSSG) [31]. The activities of both isoforms of SOD, mitochondrial (MnSOD) and cytosolic (Cu,ZnSOD), were estimated spectrophotometrically $(420 \mathrm{~nm})$ by the capacity to inhibit pyrogallol autoxidation and were expressed as $\mathrm{U}$ of superoxide dismutase/mg protein [32].

The activity of the NNT enzyme was measured in isolated liver mitochondria by direct spectrometry using a combination of previously described methods [33]. The slopes of absorbance over time were converted to mmol APAD produced/min $\times$ mg protein using the molar extinction coefficient of $5.1 \mathrm{M}^{-1} \mathrm{~cm}^{-1}$ for reduced APAD.

\subsection{Oxidative Stress in Adipose Tissue}

The retroperitoneal and inguinal fat deposits were clamped in liquid nitrogen and used for the evaluation of oxidative state, following the same protocols used for the livers. The following parameters were evaluated:
GSH content, lipid peroxidation levels and the activities of antioxidant enzymes, namely, GPx1, GR, $\mathrm{Cu}, \mathrm{ZnSOD}$ and CAT.

\subsection{Data Analysis}

The data in the figures and tables are presented as mean \pm standard error (SE) and were subjected to analysis of normality using the Shapiro-Wilk test. The experimental groups were compared using analysis of variance (ANOVA), followed by Newman-Keuls post hoc tests. The compared values are provided in the text as probability values $(p)$, and the minimum criterion of significance was $p \leq 0.05$. Statistical analyses were performed with GraphPad Prism 5.0 software (GraphPad Software Inc.).

\section{Results}

3.1 General Features, Adiposity and Adipocyte Cell Size

Sixteen weeks after the ovariectomy surgery, the OVX group presented a pronounced reduction in uterine wet weight ( $\mathrm{g} / 100 \mathrm{~g}$ BW) by approximately $73 \%$ compared to control rats $(0.068 \pm 0.012, n=7$, and $0.260 \pm 0.024, n=8$, respectively), indicating oestrogen deficiency. Treatment of the OVX rats with tibolone did not affect uterine atrophy $(0.089 \pm 0.016, n$ $=6$ ).

Fig. 1 shows the weight gain of the three animal groups studied throughout the experimental period of 16 weeks. The OVX rats exhibited higher body weight gain than control animals by approximately $31 \%$. In contrast, in the OVX rats treated with tibolone (OVX + $\mathrm{T})$, the weight gain was similar to that found in control rats. These alterations could not be attributed to differences in food ingestion (data not shown).

Fig. 2 presented the weight of four fat deposits of these animals: uterine (panel A), inguinal (panel B), retroperitoneal (panel C) and mesenteric (panel D). Although a clear increasing trend could be observed in these fat deposits, in OVX rats, only in inguinal and 


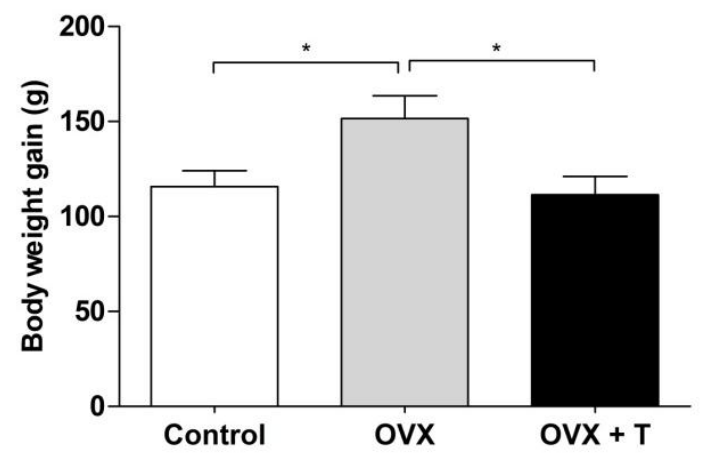

Fig. 1 Body weight gain. The body weights of sham-operated (control), bilateral ovariectomised (OVX), and OVX treated with tibolone $(\mathrm{OVX}+\mathrm{T})$ rats, measured during 16 weeks from the surgical procedures until the end of the treatment are shown. The results represent the means of the body weight gain (g) of 10 animals. Vertical bars represent the standard errors. The asterisks indicate significant differences between the values as revealed by ANOVA $(* p<0.05)$.

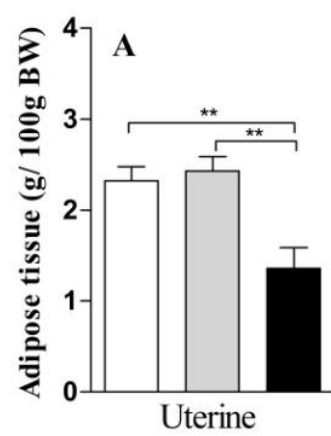

Uterine

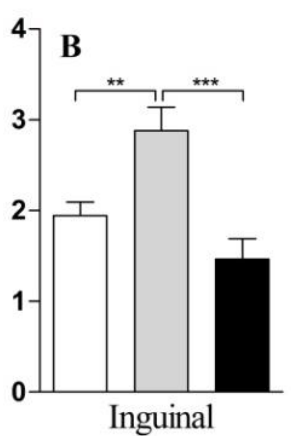

Control

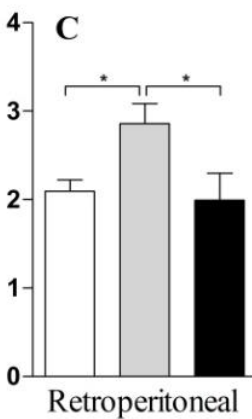

OVX
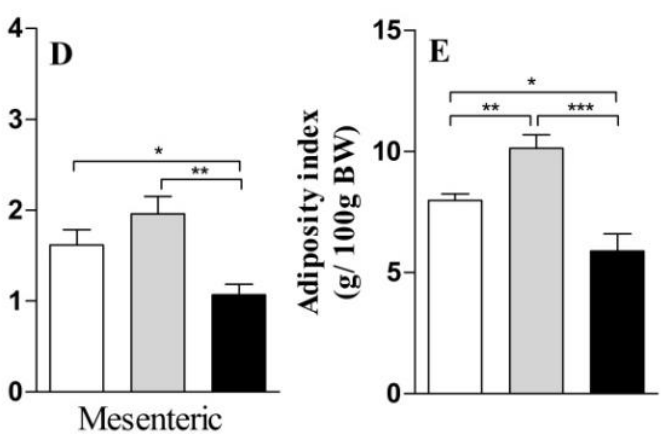

$\mathrm{OVX}+\mathrm{T}$

Fig. 2 Fat deposits weights and adiposity index. The weights of uterine (panel A), inguinal (panel B), retroperitoneal (panel C) and mesenteric (panel $D$ ) fat deposits were expressed in terms of weight per $100 \mathrm{~g}$ body weight. The adiposity index was defined as the ratio between the sums of the weights of these tissues per $100 \mathrm{~g}$ body weight (panel $\mathrm{E}$ ). The values were expressed as the means and the vertical bars represent standard errors. The asterisks indicate significant differences between the values as revealed by ANOVA $\left({ }^{*} p<0.05 ; * * p<0.01 ; * * * p<0.001\right)$.

retroperitoneal fat deposits these increases were significant $(+24$ and $+36 \%$, respectively). Tibolone administration suppressed all the changes induced by ovariectomy, and significant reductions in all fat deposit weights, including in uterine and mesenteric fats, could be detected in the OVX $+\mathrm{T}$ group. These increases in adipose tissue weights resulted in higher adiposity indexes of the OVX rats compared to the control group $(+27 \%)$, and treatment with tibolone reduced these indexes to values significantly lower than those of the control rats (Fig. 2E).

Adipocytes isolated from retroperitoneal fat deposits were used for adipocyte size analyses. Fig. 3 shows representative photomicrographs of adipocytes from control (panel A), OVX (panel B) and OVX + T (panel C) rats. The frequency distributions of adipocyte sizes of these animals are presented in panels D to F. As shown, $80 \%$ of the adipocyte areas of control rats were between 2,000 to 3,000 $\mu \mathrm{m}^{2}$ (panel D). Compared to the control group, the OVX group (panel E) exhibited a higher incidence of hypertrophic cells, presenting more than $50 \%$ of cells with an area over $3,000 \mu \mathrm{m}^{2}$. Treatment with tibolone reduced the adipocyte sizes of the OVX rats such that the curve of the frequency distribution shifted toward the distribution of the control rats (panel F). These results were corroborated by the calculation of the average cell adipocyte sizes, as presented in panel G. 

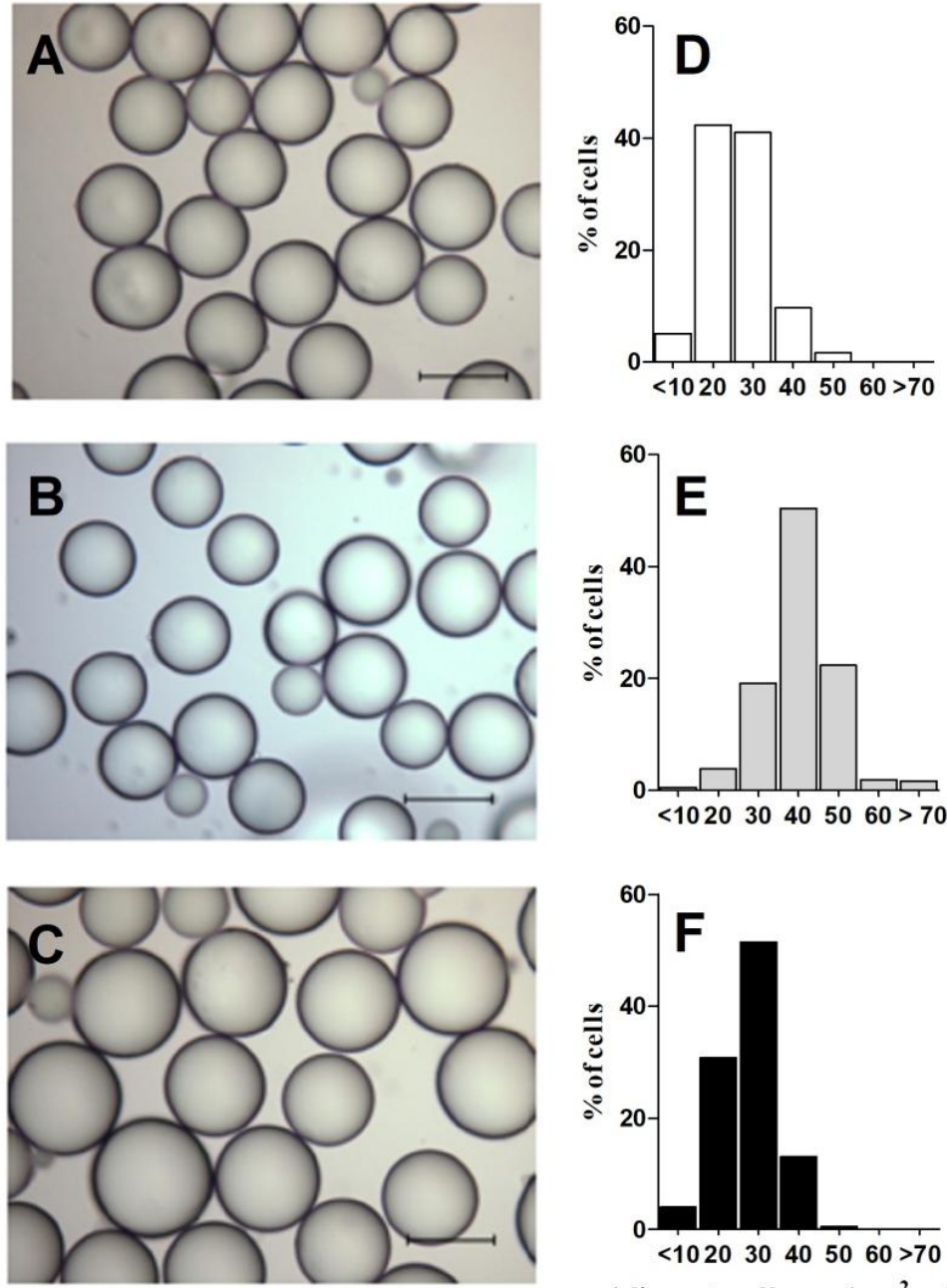

Adipocyte cell area $\left(\mu \mathrm{m}^{2} \times 100\right)$

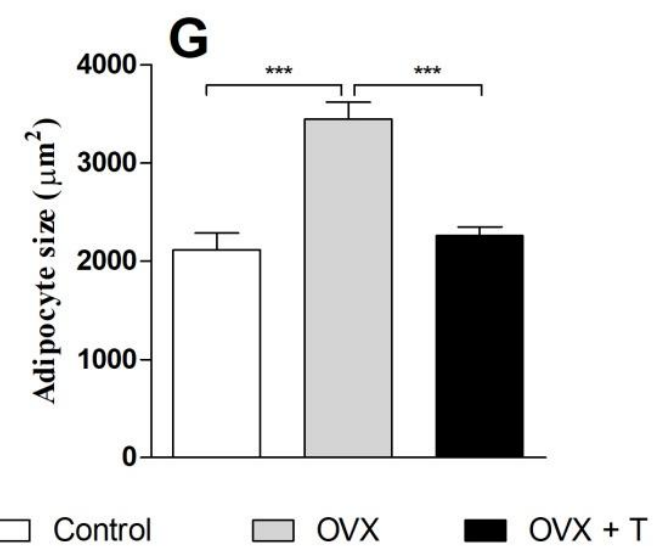

Fig. 3 The areas of retroperitoneal adipocytes. Images of adipocytes of control, OVX and OVX + T animals were captured at 40x original magnification with a high resolution digital camera (Pro-Media Series of Cibertecnics®) coupled to an optical microscope Olympus BX40®, with scale bar of $50 \mu \mathrm{m}$. Representative images of control, OVX and OVX + T rats are presented in panel $\mathrm{A}$ to $\mathrm{C}$, respectively. Adipocyte area was measured in 200 cells per rat using Image-Pro Plus 4.1® program. These measurements were used to calculate the frequency of the distribution of adipocyte sizes of control (panel D), OVX (panel E) and OVX + T (panel F) rats. In panel G, the average adipocyte sizes of these animals are presented. The values are expressed as the means and the vertical bars represent standard errors. The asterisks indicate significant differences between the values as revealed by ANOVA $(* * * p<0.001)$. 


\subsection{Blood Biochemical Dosages}

The blood biochemical analyses are presented in Table 1. The glycaemia and insulinemia of the OVX group differed significantly from those of the control rats. The peripheral insulin sensitivity was also altered in these animals, as demonstrated by the HOMA-IR index. All these undesirable alterations were completely reversed by treatment with tibolone.

The lipid profile was also altered in the OVX rats: these animals showed a significant increase in TAG, LDL cholesterol and VLDL cholesterol levels compared to control rats (increases of 38\%, 35\% and $36 \%$, respectively). However, these results were not accompanied by changes in HDL cholesterol and total cholesterol levels. Treatment of OVX rats with tibolone did not alter TAG levels compared to untreated OVX rats, which remained significantly increased by approximately $40 \%$ compared to control rats. In relation to cholesterol fractions, the $\mathrm{OVX}+\mathrm{T}$ rats presented significant reductions of total, HDL cholesterol and LDL cholesterol levels compared to the OVX group. The treatment with tibolone did not change the values of VLDL cholesterol, which was significantly increased by approximately $45 \%$ compared to control rats. NEFA was evaluated in serum but did not differ among these animal groups.

\subsection{Hormone-Sensitive Lipase (HSL) Activity of Adipose Tissue}

Fig. 4 shows HSL activity measured in the inguinal and retroperitoneal adipose tissues. Although a slight increase in HSL activity was observed in the OVX rats, only in the inguinal adipose tissue did these values reach statistical significance compared to control rats (approximately $+8 \%)$. Treatment of these animals with tibolone significantly reduced HSL activity in both adipose tissues evaluated in this work.

\subsection{Liver Lipid Content Measurements, Histochemical Analysis and Fatty Acid Oxidation}

The results of the analyses of liver lipid contents are presented in Fig. 5. In panels A to $\mathrm{C}$ are presented the representative photomicrographs of the livers from control, OVX and OVX $+\mathrm{T}$ rats, respectively. The histochemical analyses (Sudam III) show large amounts of lipid inclusions (orange) in the OVX rats, comparatively to the control and $\mathrm{OVX}+\mathrm{T}$ rats. In panel $\mathrm{D}$ are presented the results of the liver total lipid content measurements, which were increased in OVX rats, in approximately $42 \%$, comparatively to control and OVX $+\mathrm{T}$ rats, indicating extensive steatosis. The measurements of liver TAG (panel E) and cholesterol contents (panel F) revealed that among them, only TAG

Table 1 Blood biochemical analysis.

\begin{tabular}{|c|c|c|c|}
\hline & Control & OVX & $\mathrm{OVX}+\mathrm{T}$ \\
\hline Glycaemia & $101.80 \pm 2.42$ & $131.5 \pm 3.48^{* * *}$ & $105.5 \pm 3.83$ \\
\hline Insulinemia & $0.35 \pm 0.09$ & $1.75 \pm 0.21^{* * *}$ & $0.46 \pm 0.06$ \\
\hline HOMA-IR index & $2.17 \pm 0.54$ & $14.44 \pm 2.28^{* * * *}$ & $3.03 \pm 0.42$ \\
\hline Triacylglycerols & $23.59 \pm 2.04$ & $32.44 \pm 2.29^{*}$ & $32.99 \pm 2.26^{*}$ \\
\hline Total cholesterol & $63.32 \pm 3.24$ & $70.40 \pm 1.84$ & $27.47 \pm 2.10$ \\
\hline HDL-cholesterol & $33.73 \pm 2.46$ & $30.81 \pm 0.89$ & $12.88 \pm 1.39$ \\
\hline LDL-cholesterol & $24.08 \pm 1.81$ & $32.56 \pm 1.45^{* * *}$ & $9.32 \pm 1.26^{\ddagger}$ \\
\hline VLDL-cholesterol & $4.77 \pm 0.41$ & $6.49 \pm 0.46^{*}$ & $6.92 \pm 0.66^{*}$ \\
\hline NEFA & $0.68 \pm 0.05$ & $0.70 \pm 0.05$ & $0.64 \pm 0.03$ \\
\hline
\end{tabular}

Glycaemia (mg/dL; $n=6-8$ ), insulinemia (ng/dL; $n=6-8)$, HOMA-IR index $(n=6-8)$, triacylglycerols $(\mathrm{mg} / \mathrm{dL} ; n=8-9)$, total cholesterol (mg/dL; $n=8-9)$, high-density lipoprotein (HDL; mg/dL; $n=8-11)$, low-density lipoprotein (LDL; mg/dL; $n=8-11)$, very low density lipoprotein (VLDL; mg/dL; $n=10-11$ ), non-esterified fatty acids (NEFA; mmol/L; $n=7-8$ ) are expressed as the mean \pm SEM. The symbol in the same line shows the statistical significance of the values calculated by ANOVA. $* * * p<0.001$ vs. Control and OVX $+\mathrm{T} ; *^{*} p 0.05$ vs. Control; $₫ p<0.001$ vs. Control and OVX. 


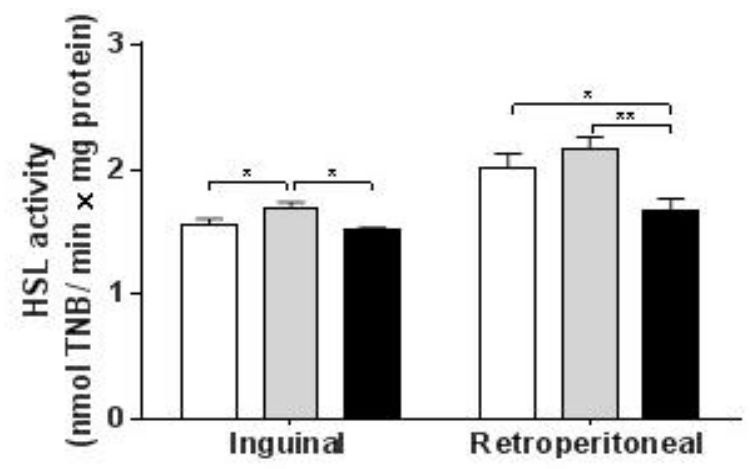

Fig. 4 The adipose tissue HSL activity. The HSL activity was evaluated in retroperitoneal and inguinal fat deposits using a lipase colorimetric assay kit. Values are expressed as the means of individual experiments with different inguinal $(n=7-8)$ or retroperitoneal $(n=7-8)$ preparations. The vertical bars represent the standard errors. The asterisks indicate significant differences between the values as revealed by ANOVA $(* p<0.05 ; * * p<0.01)$.
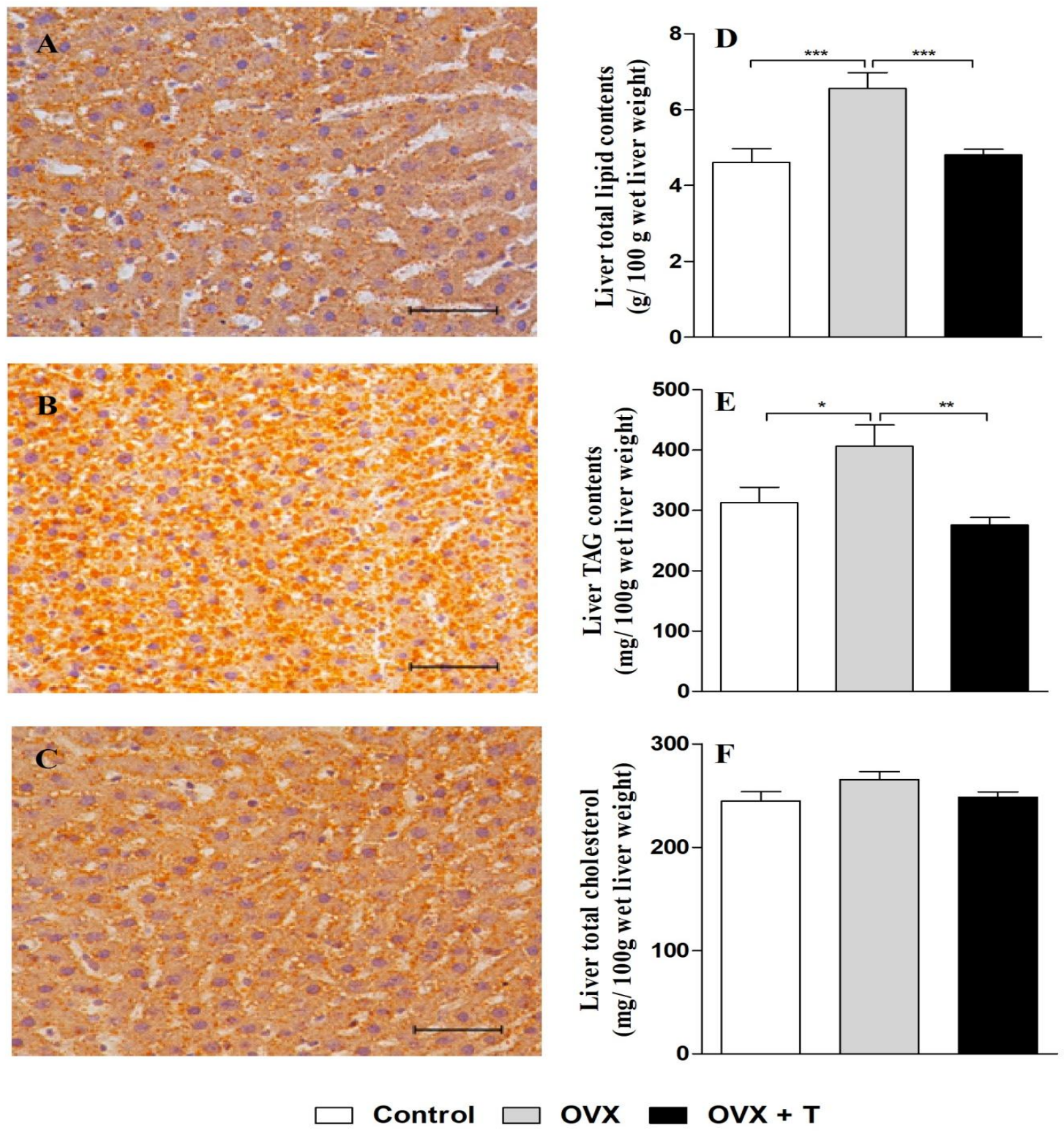
Fig. 5 Liver lipid contents and histochemical analysis. Liver fragments from control, OVX and OVX + T rats were frozen in liquid nitrogen and used for evaluation of the liver lipid contents or histochemical analysis. The representative images of histochemical analysis were captured at $40 \times$ original magnification with scale bar of $50 \mu \mathrm{m}$. In contrast to the control (image $\mathrm{A}$ ) and OVX + T (image C) rats, livers from OVX (image B) contained considerable amounts of lipid inclusions (in orange). The total lipid content of the liver was measured by gravimetry using a chloroform-methanol mixture (Panel D). The pellet was resuspended in $2 \%$ triton for quantifying the content of TAG (Panel E) and total cholesterol (Panel F) using enzymatic assay kits. The results represent the means of 10 animals and the vertical bars represent the standard errors. The asterisks indicate significant differences between the values as revealed by ANOVA $(* p<0.05 ; * * p<0.01, * * * p<0,001)$

is increased in the livers of OVX rats, in approximately $30 \%$, comparatively to control and OVX $+\mathrm{T}$ groups.

To investigate whether the fatty liver accumulation was the result of impaired $\beta$-oxidation, the fatty acid oxidation capacity of isolated mitochondria and peroxisomes were measured and the results are presented in Fig. 6. No difference was observed between the groups regarding mitochondrial $\beta$-oxidation of both octanoate and palmitate (Fig. 6A). However, peroxisomal $\beta$-oxidation of palmitate (Fig. $6 \mathrm{~B})$, which was decreased by approximately $25 \%$ in the OVX group compared to control rats, was completely restored by treatment with tibolone, and in the OVX + $\mathrm{T}$ rats, the $\beta$-oxidation capacities reached values close to those of the control group.

\subsection{Liver Redox State Parameters}

Biomarkers of oxidative stress were measured in liver homogenates and in cytosolic or mitochondrial fractions (Fig. 7, panels A to D). The mitochondrial ROS generation (Fig. 7A) was approximately 79\% higher in the OVX rats than in control rats. Treatment with tibolone significantly reduced mitochondrial ROS generation by $25 \%$, although this value remained higher than those of control rats.

The lipid peroxidation levels of the liver homogenate (Fig. 7B) and the carbonylated protein contents measured in the cytosol and in isolated mitochondria, (Fig. 7C) were all increased in the OVX rats compared to controls, but they were restored to values similar to those found in the control rats with tibolone treatment $(\mathrm{OVX}+\mathrm{T})$. The GSH contents were also evaluated in the total liver homogenates and in isolated mitochondria, and they were reduced in the OVX group in both (-26\% and $16 \%$, respectively).
Similarly, treatment with tibolone restored the GSH levels in both compartments (Fig. 7D).

In another series of experiments, the activities of antioxidant enzymes were evaluated in different liver compartments, and the results are presented in Table 2. MnSOD and GPx1 did not differ among these animals. However, the activity of mGR and NNT were significantly higher (both in approximately 33\%) in $\mathrm{OVX}+\mathrm{T}$ rats than in the control and OVX untreated groups. Among the cytosolic enzymes evaluated, GPx3 and G6PD activities were reduced in the OVX rats compared to control rats by approximately $20 \%$ and $62 \%$, respectively. Treatment with tibolone restored the G6PD activity to levels similar to those found in control rats, although it did not affect GPx3 activity. Moreover, tibolone reduced $\mathrm{Cu}, \mathrm{ZnSOD}$ activity by approximately $12 \%$ compared to the control and OVX groups. The cytosolic GR activity was affected neither by ovariectomy nor by tibolone treatment. Finally, CAT activity was significantly reduced to similar extents in the OVX and OVX $+\mathrm{T}$ rats by approximately $33 \%$ compared to the control group.

\subsection{Oxidative Stress in the Adipose Tissue}

Oxidative stress parameters were measured in the inguinal and retroperitoneal fat deposits, and the results are presented in Table 3. Contrary to the result of the redox state of the liver, no differences were observed in GSH content between the three groups of animals in either fat deposit. Lipid peroxidation levels (TBARS) were increased only in inguinal fat of OVX rats $(+23 \%)$. Therefore, with respect to the biomarkers of oxidative stress evaluated in this work, no significant change was observed in the retroperitoneal fat. 

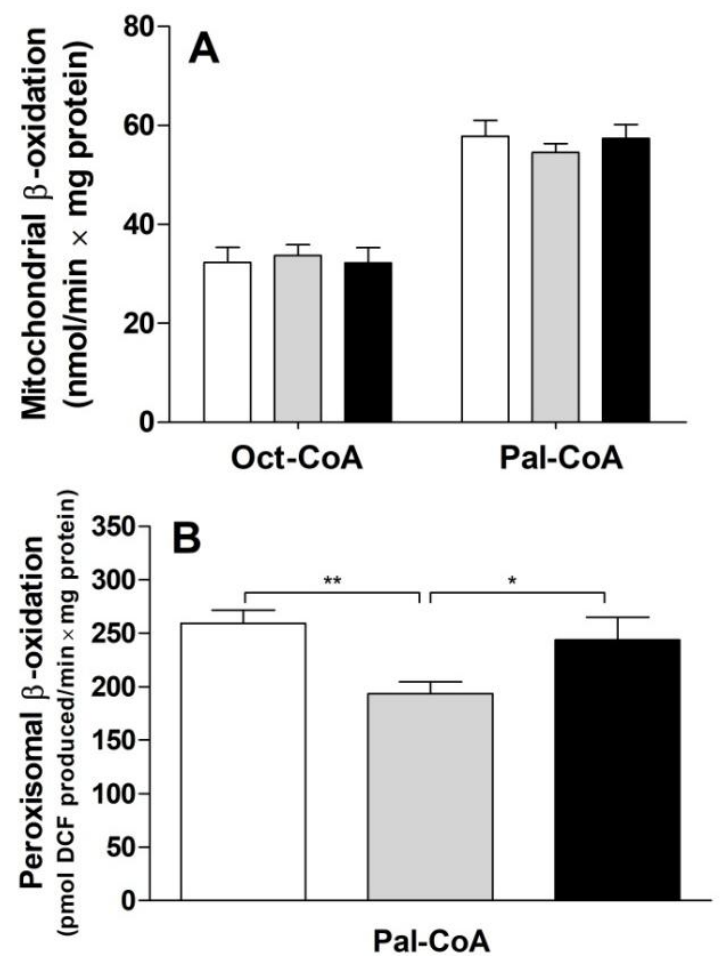

Fig. 6 Liver fatty acid $\beta$-oxidation. Liver mitochondrial fatty acid $\beta$-oxidation (Panel A) was measured by polarography in the presence of $100 \mu \mathrm{M}$ DNP. Reactions were initiated by the addition of the following: $20 \mathrm{mM}$ octanoyl-CoA $+2.0 \mathrm{mM}$ L-carnitine (Oct-CoA) or $20 \mathrm{mM}$ palmitoyl-CoA $+2.0 \mathrm{mM}$ L-carnitine (Pal-CoA). The peroxisomal fatty acyl-CoA oxidase activity (Panel B) was measured by fluorimetry. The reactions were initiated by the addition of $30 \mu \mathrm{M}$ palmitoyl-CoA (Pal-CoA). Values are expressed as the means of individual experiments with different mitochondrial $(n=9-13)$ or peroxisomal $(n=9-14)$ preparations. The vertical bars represent the standard errors. The asterisks indicate significant differences between the values as revealed by ANOVA $(* p<0.05 ; * * p<0.01)$.
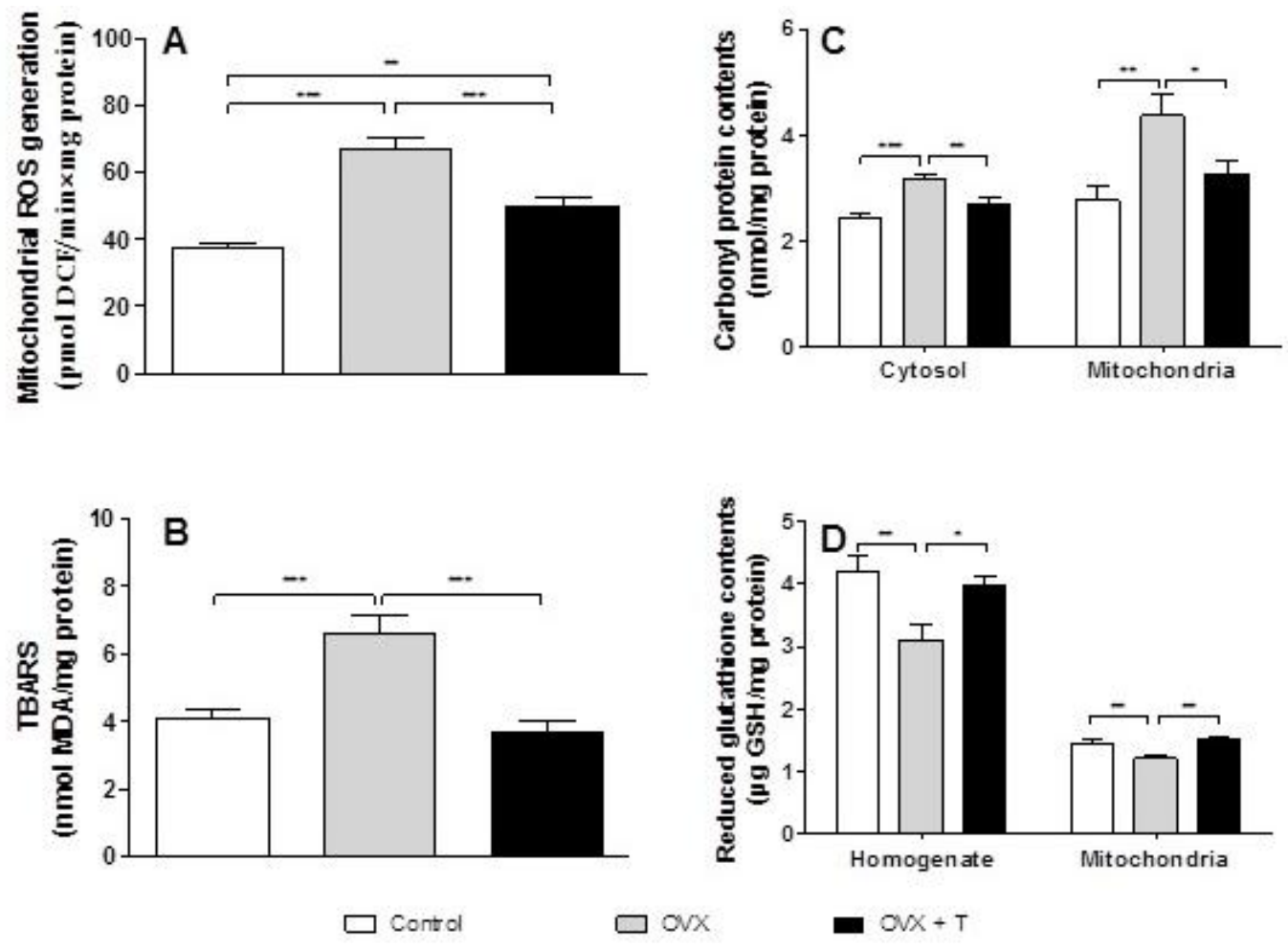
Fig. 7 Liver redox state. The mitochondrial ROS generation was evaluated by fluorimetry (Panel A; $n=5-7$ ), lipid peroxidation was measured by TBARS method (Panel $B ; n=6-8)$, the levels of carbonylated proteins were measured in the cytosol and mitochondria using by DNPH method (Panel $\mathrm{C}, n=6-8$ ), the reduced glutathione contents were evaluated in the liver homogenate and in isolated mitochondria using OPT as fluorescence probe (Panel D, $n=6-7)$. Values are expressed as the means and vertical bars represent the standard errors. The asterisks indicate significant differences between the values as revealed by ANOVA $(* p<0.05 ; * * p<0.01 ; * * * p<0.001)$.

Table 2 Effects of tibolone on liver antioxidant enzymes.

\begin{tabular}{lccc}
\hline $\begin{array}{l}\text { Enzyme activity } \\
\text { (per mg protein) }\end{array}$ & Control & OVX & OVX + T \\
\hline Mitochondria & & & $1.06 \pm 0.04$ \\
\hline MnSOD (U) & $1.06 \pm 0.07$ & $11.64 \pm 1.02$ & $15.51 \pm 1.22^{*}$ \\
mGR (nmol/min) & $11.56 \pm 0.61$ & $198.40 \pm 12.41$ & $193.20 \pm 15.41$ \\
GPx1 (nmol/min) & $222.70 \pm 18.10$ & $58.04 \pm 3.05$ & $76.59 \pm 5.14^{* *}$ \\
NNT (mmol/min) & $57.17 \pm 3.91$ & & $1.66 \pm 0.05^{* *}$ \\
\hline Cytosol & & $1.89 \pm 0.08$ & $24.09 \pm 1.16$ \\
\hline Cu, ZnSOD $(\mathrm{U})$ & $1.91 \pm 0.03$ & $24.09 \pm 1.28$ & $238.00 \pm 9.75^{\ddagger}$ \\
GR $(\mathrm{nmol} / \mathrm{min})$ & $27.20 \pm 1.48$ & $238.50 \pm 11.38^{\ddagger}$ & $28.10 \pm 2.47$ \\
GPx3 (nmol/min) & $298.50 \pm 18.89$ & $9.74 \pm 1.14^{\S}$ & $286.70 \pm 8.81^{\text {** }}$
\end{tabular}

Manganese superoxide dismutase (MnSOD; $n=7-10)$, mitochondrial glutathione reductase (mGR; $n=7$ ), glutathione peroxidase-1 (GPx1; $n=6-8)$, nicotinamide nucleotide transhydrogenase (NNT; $n=7)$, copper zinc superoxide dismutase $(\mathrm{Cu}, \mathrm{ZnSOD} ; n=10-11)$, glutathione reductase (GR; $n=9-11)$, glutathione peroxidase-3 (GPx3; $n=9-10)$, glucose 6-phosphate dehydrogenase (G6PD; $n=6$ ), catalase (CAT; $n=6$ ) are expressed as the mean \pm SEM. The symbol in the same line shows the statistical significance of the values calculated by ANOVA. ${ }^{*} p<0.05$ vs. Control and OVX; ${ }^{* *} p<0.01$ vs. Control and OVX; ${ }^{\S} p<0.001$ vs. Control and OVX + T; ${ }^{\ddagger} p<$ 0.05 vs. Control; ${ }^{\star \dagger} p<0.001$ vs. Control.

Table 3 Evaluation of oxidative stress parameters in adipose tissue.

\begin{tabular}{|c|c|c|c|}
\hline Parameter (per mg protein) & Control & OVX & $\mathrm{OVX}+\mathrm{T}$ \\
\hline \multicolumn{4}{|l|}{ Inguinal fat } \\
\hline GSH levels $(\mu \mathrm{g})$ & $1.92 \pm 0.16$ & $2.05 \pm 2.05$ & $2.04 \pm 0.22$ \\
\hline TBARS (nmol) & $18.32 \pm 1.34$ & $22.50 \pm 0.92^{\S}$ & $17.11 \pm 1.62$ \\
\hline $\mathrm{Cu}, \mathrm{ZnSOD}(\mathrm{U})$ & $1.55 \pm 0.04$ & $2.70 \pm 0.22^{*}$ & $2.95 \pm 0.25^{*}$ \\
\hline GR (nmol/min) & $8.17 \pm 0.82$ & $10.93 \pm 1.09$ & $12.33 \pm 1.03^{*}$ \\
\hline GPx3 (nmol/min) & $49.54 \pm 3.63$ & $61.58 \pm 3.98^{*}$ & $80.03 \pm 4.49^{* *}$ \\
\hline $\mathrm{CAT}(\mu \mathrm{mol} / \mathrm{min})$ & $104.70 \pm 7.03$ & $111.50 \pm 7.92$ & $123.40 \pm 10.06$ \\
\hline \multicolumn{4}{|l|}{ Retroperitoneal fat } \\
\hline GSH levels $(\mu \mathrm{g})$ & $2.24 \pm 0.16$ & $2.25 \pm 0.22$ & $2.45 \pm 0.11$ \\
\hline TBARS (nmol) & $15.34 \pm 0.24$ & $14.27 \pm 0.49$ & $14.39 \pm 0.43$ \\
\hline $\mathrm{Cu}, \mathrm{ZnSOD}(\mathrm{U})$ & $1.88 \pm 0.13$ & $1.74 \pm 0.09$ & $1.50 \pm 0.13$ \\
\hline GR (nmol/min) & $16.82 \pm 2.10$ & $13.94 \pm 0.93$ & $17.04 \pm 1.13$ \\
\hline GPx3 (nmol/min) & $87.07 \pm 12.80$ & $73.93 \pm 7.69$ & $72.86 \pm 7.13$ \\
\hline $\mathrm{CAT}(\mu \mathrm{mol} / \mathrm{min})$ & $149.60 \pm 5.20$ & $171.70 \pm 6.34$ & $167.20 \pm 10.94$ \\
\hline
\end{tabular}

Copper zinc superoxide dismutase (Cu, ZnSOD; $n=6-9)$, glutathione reductase (GR; $n=7-9)$, glutathione peroxidase-3 (GPx3; $n=$ 9-11), catalase (CAT; $n=7-9$ ) are expressed as the mean \pm SEM. The symbol in the same line shows the statistical significance of the values calculated by ANOVA. ${ }^{\star} p<0.05$ vs. Control; ${ }^{\star} p<0.001$ vs. Control; $* * p<0.01$ vs. Control and OVX; ${ }^{\circledR} p<0.05$ vs. Control and OVX $+\mathrm{T}$. 
Among the antioxidant enzymes evaluated in the inguinal fat deposit, $\mathrm{Cu}, \mathrm{ZnSOD}$ was significantly increased by approximately $74 \%$ in the OVX rats compared to control rats. However, the OVX $+\mathrm{T}$ group did not differ from untreated OVX rats, which remained increased compared to control rats. Progressive and significant increases in GPx3 activity were observed between control rats and both OVX and $\mathrm{OVX}+\mathrm{T}$ rats. In addition, CAT activity in the inguinal adipose tissue did not differ among these three animal groups, but GR activity was significantly increased in the OVX $+\mathrm{T}$ group compared to the control and OVX groups.

\section{Discussion}

The results of the present study revealed that, 16 weeks after ovariectomy, rats presented uterine atrophy and developed several characteristics of MS, including increased adiposity associated with adipocyte hypertrophy, peripheral IR, dyslipidaemia and NAFLD (Fig. 8). Liver fat accumulation was followed by a worsening of the redox state, as could be demonstrated by the increases in ROS generation, lipid peroxidation and carbonyl protein contents. However, the increase in the fat deposits did not reflect impacts on redox state, again demonstrating that ectopic fat accumulation exerts more damaging cellular effects [34].

OVX rats exhibited increases in fat deposits and hypertrophy of adipocytes. Tibolone reversed all these conditions, reducing the fat deposits and adipocyte sizes. Our results are in accordance with a previous study in which it was demonstrated that daily administration of tibolone (2 $\mathrm{mg} / \mathrm{kg})$ to OVX-aromatase knockout (ArKO) mice resulted in an obvious decrease in the adipose phenotype, reducing adipocyte size [35].

It has long been known that the hypertrophy of adipocytes leads to important cellular dysfunctions, including overproduction of TNF- $\alpha$ [5, 7], and higher expression of TNF- $\alpha$ [36, 37], which induces IR in both adipocytes and myocytes [8]. In this way, one important mode of action of tibolone was probably the reduction in the visceral adipocyte size, as demonstrated here and previously described for postmenopausal women [38].

The OVX rats also presented extensive NAFLD, which has been considered as an IR-syndrome. In insulin resistant adipocytes, the inhibition of HSL enzyme is suppressed and the lipolysis increases [39]. The increased release of free fatty acids directly into the portal vein overloads the liver, inducing NAFLD [40]. In the present work, an increase in the HSL enzyme activity was, in fact, observed in the OVX rats, a condition that was reversed by the treatment with tibolone.

Besides, the increased delivery of fatty acids to hepatocytes is sufficient to induce hepatic IR [41] and the inhibition of gluconeogenesis by insulin is impaired thus resulting in hyperglycemia, at the same time that put more substrate available to hepatic FA synthesis

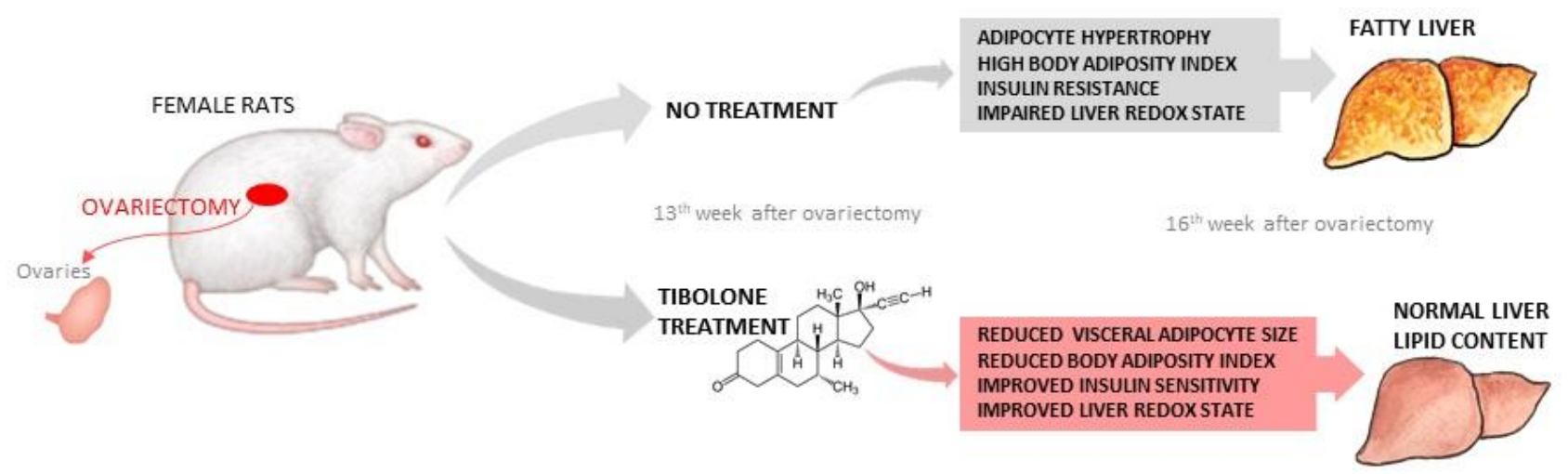

Fig. 8 Effects of tibolone treatment in ovariectomised rats. 
$[42,43]$. This was other finding in the current work: the OVX rats were hyperglycaemic, a condition that was also reversed by tibolone.

However, IR could trigger NAFLD by other mechanisms, beyond the increased lipolysis. In the liver, insulin robustly promotes liver FA synthesis and decreases FA oxidation. In fact, the peroxisomal $\beta$-oxidation was impaired in OVX rats. Therefore, the liver fat accumulation could be attributed, at least in part, to a reduction in this pathway. Similarly, the increase in peroxisomal $\beta$-oxidation induced by treatment with tibolone could be another contributing factor to the improvement of steatosis in these animals. The oestrogen deficiency could also be involved in the reduction in the peroxisomal $\beta$-oxidation $[11,44]$ and, in this sense, tibolone could be exerting oestrogenic actions.

Recent studies performed in our laboratory revealed for the first time that tibolone was also effective in reversing NAFLD in hypertensive, OVX rats [45], by reducing the blood arterial pressure. So, although NAFLD has been considered, for a long time, as a IR-syndrome, recent studies have noted the importance of hypertension as a triggering factor of NAFLD [46, 47]. In this regard, it should be noted that in those animals, NAFLD arose very early after the ovariectomy surgery (3 weeks), and independent on the IR, which was absent $[15,45,48]$. Thus, tibolone has been proven to be effective in reversing steatosis associated with IR, as evidenced in this work, as well as to be associated with hypertension, as previously demonstrated by us [45].

With respect to the lipid profile, tibolone exerted the undesirable effect of lowering HDL cholesterol levels, as described before [14, 49]. The mechanisms by which tibolone exerts this effect are uncertain, but they could involve androgenic actions of the tibolone metabolites or a more rapid HDL clearance [50, 51]. Furthermore, similar to oestrogen [52], the oral administration of tibolone leads to increases in the bile concentrations of cholesterol and trihydroxy acids [53], and this could also contribute to the observed reductions in HDL levels.

However, tibolone was also effective in reducing LDL levels and, in doing this, restored the HDL/LDL ratio. This index of cardiovascular risk, which was significantly reduced in the OVX rats $(0.996 \pm 0.061)$, returned to values close to those found in control rats $(1.409 \pm 0.147)$ in the OVX $+\mathrm{T}$ rats $(1.370 \pm 0.048)$, which implies in a cardiovascular protective action. These alterations in the lipid profile were also accompanied by reductions in lower total cholesterol levels without interference in the plasmatic TAG levels, and the results agree with those described by Henriques et al. [14].

The impacts of oestrogen deficiency on the oxidative state of the liver and adipose tissues (retroperitoneal and inguinal) were also investigated. Oestrogen is a powerful antioxidant [54], and thus, the oestrogen deficiency has been associated with disturbances in redox state in OVX rats $[48,55]$ and postmenopausal women [56].

Among the effects of tibolone in the liver redox state, the increases in GSH levels stand out, which were reduced in both the liver homogenate and isolated mitochondria of the OVX rats and were restored by treatment with tibolone. These results agree with previous studies performed by our team $[45,48,55]$ and could be largely attributed to increases in mitochondrial ROS generation in the OVX rats $[45,48$, 57]. Moreover, oxidative damage in the liver was also evidenced in the OVX rats by the higher levels of lipid peroxidation and carbonylated protein contents. Similar to oestrogen, tibolone improved the redox state of the liver by reducing ROS generation, as well as, the oxidative damage associated, as demonstrated by reduction of the lipid peroxidation and carbonylated protein levels.

Alterations in the activities of antioxidant enzymes, especially those involved in the GSH cycle, could also contribute to oxidative damage. In this respect, among the observed alterations, a factor could contribute to the 
worsening of the liver redox state of OVX rats and its improvement by treatment with tibolone, namely, the reduction of G6PD and GR activities in OVX rats and its restoration upon treatment with tibolone. These results are expected, as it has long been known that G6PD is activated by oestrogens [58], and its reduced activity in OVX rats was already described by us [45, $48,55]$. In addition, in mitochondria, the activities of NNT were also increased by tibolone. The NNT enzyme has also been associated with IR [59], and the importance of the increase in its activity by tibolone should not be minimized. Therefore, the overall effects of treatment with tibolone in the redox state of the liver were beneficial. Even a reduction in the activity of $\mathrm{Cu}, \mathrm{ZnSOD}$ was expected in the liver of treated rats, as the expression of this enzyme is controlled by the presence of ROS [60].

As evidenced in this work, the redox state of the retroperitoneal adipose tissue was not altered by oestrogen deficiency or by tibolone treatment, whereas the inguinal adipose tissue showed higher lipid peroxidation levels in the OVX rats. In agreement with this, differences have been described between visceral and subcutaneous fat deposits with respect to their antioxidant state and responses [34]. Treatment with tibolone also showed beneficial effects in the redox state of the inguinal adipose tissue, as could be detected by the reduction in TBARS levels. The alterations observed in the activities of $\mathrm{Cu}, \mathrm{ZnSOD}$ and GPx3 of inguinal adipose tissue were likely adaptive.

In summary, the metabolic alterations induced by ovariectomy resulted in the development of obesity, visceral adipocyte hypertrophy, IR and NAFLD, which was associated with a decline in the liver redox state but not in the adipose tissue. On the other hand, tibolone reduced the adiposity and adipocyte size, which was accompanied by improvements in several metabolic disorders, including IR and NAFLD. One important mode of action of tibolone was to reduce the visceral adipocyte size and these results reinforce the relationship between visceral adipocyte size, IR and
NAFLD. Some actions of tibolone, however, could involve the binding to oestrogen receptors, in especial those antioxidant effects.

\section{Acknowledgements}

This work was supported by grants from Coordenação de Aperfeiçoamento de Pessoal de Nível Superior (CAPES).

\section{Declarations of Interest}

None.

\section{References}

[1] Carr, M. C. 2003. "The Emergence of the Metabolic Syndrome with Menopause." J Clin Endocrinol Metab 88: 2404-11.

[2] Grattagliano, I., Palmieri, V. O., Portincasa, P., Moschetta, A., and Palasciano, G. 2008. "Oxidative Stress-Induced Risk Factors Associated with the Metabolic Syndrome: A Unifying Hypothesis." J Nutr Biochem 19: 491-504.

[3] Diehl, A. M. 2004. "Tumor Necrosis Factor and Its Potential Role in Insulin Resistance and Nonalcoholic Fatty Liver Disease." Clin Liver Dis 8: 619-38.

[4] Skurk, T., Alberti-Huber, C., Herder, C., and Hauner H. 2007. "Relationship between Adipocyte Size and Adipokine Expression and Secretion." J Clin Endocrinol Metab 92: 1023-33.

[5] O'Connell, J., Lynch, L., Cawood, T. J., et al. 2010. "Relationship of Omental and Subcutaneous Adipocyte Size to Metabolic Disease in Severe Obesity." PloS One 5: e9997.

[6] Altomonte, J., Harbaran, S., Richter, A., and Dong, H. 2003. "Fat Depot-Specific Expression of Adiponectin Is Impaired in Zucker Fatty Rats." Metabolism 52: 958-63.

[7] Winkler, G., Kiss, S., Keszthelyi, L., et al. 2003. "Expression of Tumor Necrosis Factor (TNF)-Alpha Protein in the Subcutaneous and Visceral Adipose Tissue in Correlation with Adipocyte Cell Volume, Serum TNF-Alpha, Soluble Serum TNF-Receptor-2 Concentrations and C-Peptide Level." Eur J Endocrinol 149: 129-35.

[8] Salans, L. B., Knittle, J. L., and Hirsch, J. 1968. “The Role of Adipose Cell Size and Adipose Tissue Insulin Sensitivity in the Carbohydrate Intolerance of Human Obesity." J Clin Invest 47: 153-65.

[9] Tilg, H., and Hotamisligil, G. S. 2006. "Nonalcoholic Fatty Liver Disease: Cytokine-Adipokine Interplay and Regulation of Insulin Resistance.” Gastroenterology 131: 934-45. 
[10] Gaspard, U. 2009. "Hyperinsulinaemia, a Key Factor of the Metabolic Syndrome in Postmenopausal Women." Maturitas 62: 362-5.

[11] D’Eon, T. M., Souza, S. C., Aronovitz, M., Obin, M. S., Fried, S. K., and Greenberg, A. S. 2005. "Estrogen Regulation of Adiposity and Fuel Partitioning." J Biol Chem 280: 35983-91.

[12] Grodstein, F., Manson, J. E., and Stampfer, M. J. 2006. "Hormone Therapy and Coronary Heart Disease: The Role of Time Since Menopause and Age at Hormone Initiation." $J$ Womens Health (Larchmt) 15: 35-44.

[13] Hänggi, W., Lippuner, K., Jaeger, P., Birkhäuser, M. H., and Horber, F. F. 1998. "Differential Impact of Conventional Oral or Transdermal Hormone Replacement Therapy or Tibolone on Body Composition in Postmenopausal Women." Clin Endocrinol (Oxf) 48: 691-9.

[14] Henriques, H. N., Câmara, N. R., de Carvalho, A. C., Pantaleão, J. A., and Guzmán-Silva, M. A. 2010. "Effect of High Doses of Tibolone in Body Weight and Lipid Profile of Ovariectomized Rat." Rev Bras Ginecol Obstet 32: 88-93.

[15] Paquette, A., Shinoda, M., Rabasa-Lhoret, R., Prud'homme, D., and Lavoie, J. M. 2007. “Time Course of Liver Lipid Infiltration in Ovariectomized Rats: Impact of a High-Fat Diet." Maturitas 58: 182-90.

[16] Matthews, D. R., Hosker, J. P., Rudenski, A. S., Naylor, B. A., Treacher, D. F., and Turner, R. C. 1985. "Homeostasis Model Assessment: Insulin Resistance and Beta-Cell Function from Fasting Plasma Glucose and Insulin Concentrations in Man.” Diabetologia 28: 412-9.

[17] Rodbell, M. 1964. "Metabolism of Isolated Fat Cells. Effects of Hormones on Glucose Metabolism and Lipolysis." J Biol Chem. 239: 357-80.

[18] Folch, J., Lees, M., and Sloane-Stanley, G. H. 1957. “A Simple Method for the Isolation and Purification of Total Lipids from Animal Tissues." J Biol Chem 226: 497-509.

[19] Bracht, A., Ishii-Iwamoto, E. L., Salgueiro-Pagadigorria, C. L. 2003. "Técnica de centrifugação e fracionamento celular." In Métodos de Laboratório em Bioquímica, edited by Bracht, A., Ishii-Iwamoto, E. L. São Paulo, Brazil: Manole Ltda, 77-101.

[20] Natarajan, S. K., Eapen, C. E., Pullimood, A. B., and Balasubramanian, K. A. 2006. "Oxidative Stress in Experimental Liver Microvesicular Steatosis: Role of Mitochondria and Peroxisomes." J Gastroenterol Hepatol 21: 1240-9.

[21] Lowry, O. H., Rosebrough, N. J., Farr, A. L., and Randall, R. J. 1951. "Protein Measurement with the Folin Phenol Reagent." J Biol Chem 193: 265-75.

[22] Garland, P. B., Shepherd, D., Nicholls, D. G., Yates, D.
W., and Light, P. A. 1969. "Interactions between Fatty Acid Oxidation and the Tricarboxylic Acid Cycle." In Citric Acid Cycle, edited by Lowestein J. M. New York, NY: Marcel Dekker, 163-212.

[23] Small, G. M., Burdett, K., and Connock, M. J. 1985. “A Sensitive Spectrophotometric Assay for Peroxisomal Acyl-CoA Oxidase." Biochem J 227: 205-10.

[24] Zaccagnino, P., Saltarella, M., D’oria, S., Corcelli, A., Saponetti, M. S., and Lorusso, M. 2009. "N-Arachidonylglycine Causes ROS Production and Cytochrome c Release in Liver Mitochondria." Free Radic Biol Med 47: 585-92.

[25] Hissin, P. J., and Hilf, R. 1976. "A Fluorometric Method for Determination of Oxidized and Reduced Glutathione in Tissues." Anal Biochem 74: 214-26.

[26] Hermoso, D. A. M, Campos-Shimada, L. B., Gilglioni, E. H., et al. 2016. "Melatonin Protects Female Rats against Steatosis and Liver Oxidative Stress Induced by Oestrogen Deficiency." Life Sciences 157: 178-86.

[27] Ohkawa, H., Ohishi, N. and Yagi, K. 1979. "Assay for Lipid Peroxides in Animal Tissues by Thiobarbituric Acid Reaction." Anal Biochem 95: 351-8.

[28] Aebi, H. 1974. "Catalase." In Methods of Enzymatic Analysis, edited by Bergmeyer, H. U. New York: Academic Press, 673-90.

[29] Tian, W. N., Pignatare, N., and Stanton, R. C. 1994. "Signal Transduction Proteins That Associate with the Platelet-Derived Growth Factor (PDGF) Receptor Mediate the PDGF-Induced Release of Glucose-6-Phosphate Dehydrogenase from Permeabilized Cells." J Biol Chem 269: 14798-805.

[30] Paglia, E. D., and Valentine, W. N. 1967. "Studies on the Quantitative and Qualitative Characterization of Erythrocyte Glutatione Peroxidase." J Lab Clin Med 70: 158-69.

[31] Mize, C. E., and Langdon, R. G. 1962. "Hepatic Glutathione Reductase. Purification and General Kinetic Properties." J Biol Chem 237: 1589-95.

[32] Marklund, S., and Marklund, G. 1974. "Involvement of the Superoxide Anion Radical in the Autoxidation of Pyrogallol and a Convenient Assay for Superoxide Dismutase." Eur J Biochem 47: 469-74.

[33] Ronchi, J. A., Figueira, T. R., Ravagnani, F. G., Oliveira, H. C., Vercesi, A. E., and Castilho, R. F. 2013. "A Spontaneous Mutation in the Nicotinamide Nucleotide Transhydrogenase Gene of C57BL/6J Mice Results in Mitochondrial Redox Abnormalities." Free Radic Biol Med 63: 446-56.

[34] Jankovic, A., Korac, A., Srdic-Galic, B., et al. 2014. "Differences in the Redox Status of Human Visceral and Subcutaneous Adipose Tissues-Relationships to Obesity and Metabolic Risk.” Metabolism 63: 661-71. 
[35] Van Sinderen, M. L., Boon, W. C., Ederveen, A. G. H., et al. 2009. "The Estrogenic Component of Tibolone Reduces Adiposity in Female Aromatase Knockout Mice." Menopause 16: 582-8.

[36] Hotamisligil, G. S., and Shargill, N. S. 1993. "Adipose Expression of Tumor Necrosis Factor: A Direct Role in Obesity-Linked Insulin Resistance." Science 259: 87-1.

[37] Kern, P. A., Saghizaheh, M., Ong, J. M., Bosch, R. J., Deem, R., and Simsolo, R. B. 1995. "The Expression of Tumor Necrosis Factor in Human Adipose Tissue. Regulation by Obesity, Weight Loss, and Relationship to Lipoprotein Lipase." J Clin Invest 95: 2111-9.

[38] Carranza-Lira, S., Bárcena-Jacobo, T. D., Sandoval-Barragán, M. P., and Ramos-León, J. C. 2011. "Visceral Adiposity after Tibolone Use." Int J Gynecol Obstet 15: 191-3.

[39] Gabriely, I., and Barzilai, N. 2003. "Surgical Removal of Visceral Adipose Tissue: Effects on Insulin Action". Curr Diab Rep 3: 201-6.

[40] Item, R., and Konrad, D. 2012. "Visceral Fat and Metabolic Inflammation: The Portal Theory Revisited." Obesity Reviews 13: 30-9.

[41] Kim, J. K., Fillmore, J. J., Chen, Y., et al. 2001. "Tissue-Specific Overexpression of Lipoprotein Lipase Causes Tissue-Specific Insulin Resistance." Proc Natl Acad Sci USA 98: 7522-7.

[42] Kotronen, A., Westerbacka, J., Bergholm, R., et al. 2007. "Liver Fat in the Metabolic Syndrome." J Clin Endocrinol Metab 92 (9): 3490-7.

[43] Vanni, E., Bugianesi, E., Kotronen, A., et al. 2010. "From the Metabolic Syndrome to NAFLD or Vice Versa?" Digestive and Liver Disease 42: 320-30.

[44] Paquette, A., Wang, D., Jankowski, M., Gutkowska, J., and Lavoie, J. M. 2008. "Effects of Ovariectomy on PPAR Alpha, SREBP-1c, and SCD-1 Gene Expression in the Rat Liver." Menopause 15: 1169-75.

[45] Gilglioni, E. H., Campos, L. B., Oliveira, M. C., et al. 2013. "Beneficial Effects of Tibolone on Blood Pressure and Liver Redox Status in Ovariectomized Rats with Renovascular Hypertension." J Gerontol A Biol Sci Med Sci 68: 510-20.

[46] Lau, K., Lorbeer, R., Haring, R., et al. 2010. "The Association between Fatty Liver Disease and Blood Pressure in a Population-Based Prospective Longitudinal Study." J Hypertens 28: 1829-35.

[47] Lopez-Suarez, A., Guerrero, J. M., Elvira-Gonzalez, J., Beltran-Robles, M., Canas-Hormigo, F., Bascunana-Quirell, A. 2011. "Nonalcoholic Fatty Liver Disease Is Associated with Blood Pressure in Hypertensive and Nonhypertensive Individuals from the General Population with Normal Levels of Alanine Aminotransferase." Eur J Gastroenterol Hepatol 23:
1011-7.

[48] Campos, L. B., Gilglioni, E. H., Garcia, R. F., et al. 2012. "Cimicifuga racemosa Impairs Fatty Acid $\beta$-Oxidation and Induces Oxidative Stress in Livers of Ovariectomized Rats with Renovascular Hypertension." Free Radic Biol Med 53: 680-9.

[49] Vassalle, C., Cicinelli, E., Lello, S., Mercuri, A., Battaglia, D., and Maffei, S. 2011. "Effects of Menopause and Tibolone on Different Cardiovascular Biomarkers in Healthy Women." Gynecol Endocrinol 27: 163-9.

[50] von Eckardstein, A., Nofer, J. R., and Assmann, G. 2001. "High Density Lipoproteins and Arteriosclerosis: Role of Cholesterol Efflux and Reverse Cholesterol Transport." Arterioscler Thromb Vasc Biol 21: 13-27.

[51] Mikkola, T. S., Anthony, M. S., Clarkson, T. B., and St. Clair, R. W. 2002. "Serum Cholesterol Efflux Potential in Postmenopausal Monkeys Treated with Tibolone or Conjugate Estrogens.” Metabolism 51: 523-30.

[52] Uhler, M. L., Marks, J. W., and Judd, H. L. 2000. "Estrogen Replacement Therapy and Gallbladder Disease in Postmenopausal Women.” Menopause 7: 162-7.

[53] Czerny, B., Teister, M., Juzyszyn, Z., Mysliwiec, Z., and Pawlik, A. 2005. "Effect of Tibolone on Turnover of Cholesterol to Bile Acids in Ovariectomized Rats." Menopause 12: 609-12.

[54] Behl, C., Skutella, T., Lezoualc'h, F., et al. 1997. "Neuroprotection against Oxidative Stress by Estrogens: Structure-Activity Relationship." Mol Pharmacol 51: 535-41.

[55] Moreno, F. N., Campos-Shimada, L. B., da Costa, S. C., et al. 2015. "Vitex agnus-castus L. (Verbenaceae) Improves the Liver Lipid Metabolism and Redox State of Ovariectomized Rats." Evid Based Complement Alternat Med. 2015: 212378.

[56] Doshi, S. B. and Agarwal, A. 2013. "The Role of Oxidative Stress in Menopause." J Midlife Health 4: 140-6.

[57] Borras, C., Gambini, J., and Vina, J. 2007. "Mitochondrial Oxidant Generation Is Involved in Determining Why Females Live Longer Than Males." Front Biosci 12: 1008-13.

[58] Ibim, S. E. M., Randall, R., Han, P., and Musey, P. 1989. "Modulation of Hepatic Glucose-6-Phosphate Dehydrogenase Activity in Male and female Rats by Estrogen." Life Sci 45: 1559-65.

[59] Nicholson, A., Reifsnyder, P. C., Malcom, R., et al. 2010. "Diet Induced Obesity in Two C57BL/6 Substrains with Intact or MUTANT NICOTINAMIDe Nucleotide Transhydrogenase (Nnt) Gene." Obesity (Silver Spring) 18 (10): 1902-5.

[60] Scott, M. D., Meshnick, S. R., and Eaton, J. W. 1987. "Superoxide Dismutase-Rich Bacteria. Paradoxical Increase in Oxidant Toxicity." J. Biol. Chem. 15: 3640-5. 